\title{
Multi-Scale Compressed Ghost Imaging System
}

\author{
Zhang $\mathrm{Na}^{1, *}$, Gan Quan ${ }^{2}$ \\ ${ }^{1}$ Institute of Computer Science and Engineering of Henan University and Urban Construction, Pindingshan, Henan, \\ 467036, China; ${ }^{2}$ College of Computer Science and Technology, Pingdingshan University, Pingdingshan, Henan 467000, \\ China
}

\begin{abstract}
Fixed-scale speckles are used in existing ghost imaging system, while the current instrument can achieve multiscale speckles ghost imaging. This paper focuses on the research of compressed sensing ghost imaging system using multi-scale speckles. Through the computer simulation and using the four resolution scales image as the original image, restored images are obtained to analysis on a variety of different scales and different proportion of speckles cases. The results show that the compressed sensing ghost imaging system using multi-scale speckles can get closer to the true value of image. And when the small scale speckles occupy large proportion, the recovered image resolution is not disturbed.
\end{abstract}

Keywords: Compressed sensing, ghost imaging, multi-scale speckles.

\section{INTRODUCTION}

Ghost imaging as a new imaging system attracts extensive attention worldwide by domestic and overseas scholars [1-6]. The light source modulation was realized in the ghost imaging system to obtain the high resolution imaging of single pixel of object by the related algorithm At present, Accurate phase modulator parts of digital micro reflection lens is used by the light modulator to obtain the light intensity distribution of objects through the calculation method of light transmission by the use of modem information. Single pixel detector can detect where the area-array detector can't response, it can offset the shortage of the area-array and enrich the detect band of imaging system, it has a certain application prospect and value. British scientist has build up the three-dimension ghost imaging system using the algorithm from multiple perspective, and carry on the face high resolution $3 \mathrm{~d}$ imaging, The results of the study is published in Science, and is highly appreciated by domestic and foreign scholars. Ghost imaging system based on polarization detection is proposed by the Anhui Institute of OpticsFine Mechanics, China. The system can classify objects detection identification. Single pixel detector has the advantages of high temporal resolution for high-precision threedimensional imaging. British researchers do the experimental research by use of this feature in the indoor and get threedimensional images of the objects [4]. Three-dimensional ghost imaging technology is realized by the pulse time sequence at Shanghai Institute of OpticsFine Mechanics, China, they gained the image which has the lateral resolution of $2 \mathrm{~cm}$ and the longitudinal resolution of $60 \mathrm{~cm}$ at one thousand metres. At present, Related research has already covers all aspects of the ghost imaging system, and the theoretical system has been built up perfectly [1].
In order to obtain the image in the ghost imaging system, we often use correlation algorithm to recover, But the disadvantage is that it requires more hits and the signal-to-noise ratio of the image is low. At present, the compression sensing algorithm in ghost imaging has been widely used in the ghost imaging system, this algorithm can not only effectively reduce the sampling number but also improve the image resolution and signal-to-noise ratio. The effectiveness of the algorithm is confirmed by a large amount of theoretical and experimental research [1]. Ghost imaging system used in the scale of the speckle intensity distribution is a constant. According to the analysis of literature [1], The scale of the speckle has a decisive influence on the the image resolution and the signal-to-noise ratio. Now, with the development of high precision optical modulator, it is easy to implement the different scales of speckle. The present study, however, does not study of multi-scale speckle ghost imaging system, This paper will study on this content.

\section{MULTI-SCALE GHOST IMAGING SYSTEM}

Fig. (1) shows the Multi-Scale imaging system. The light of laser light source exit via the phase modulation system, then go through the polarization state of generator, generate specific polarization of light to illuminate the object, The reflected light is divided into two orthogonal polarization state beam respectively by two single pixel detector for intensity information after polarization analysis system. The difference between the system and the conventional ghost imaging is that it use the controllable optical modulation system, and that it can be obtained by calculation to get exposure speckle distribution form of the object, Moreover it can get the different scales of speckle distribution through the control of the optical modulation system. According to the results of theoretical analysis of literature [1], it is known that the resolution ratio of the image in the ghost imaging system is determined by speckle scale, the smaller speckle scale, the higher the resolution of the image object. But at 


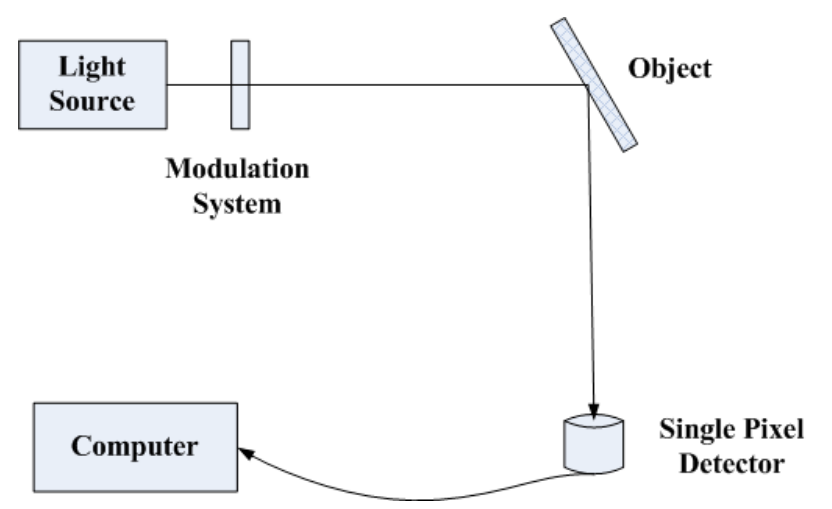

Fig. (1). Sketch-map of multi-scale ghost imaging system.

the same time the object image signal-to-noise ratio is low. In fact the objects in the image is composed of different scales of resolution, By the nature of Fourier transform, we can also get the same conclusion. At present, the unified speckle measure is used to restore the image object in the most of the ghost imaging system. Therefore, it need to analyze the the different speckle measurement results when we restore the same object images. We assume the Nth sampling intensity $I_{B}^{(n)}$ can be expressed as:

$\sum_{x} I^{(n)}(x, l) G(x)=I_{B}^{(n)}$

where $G(x)$ is the strength information of the object or the polarization information. $I^{(n)}(x, l)$ is Nth speckle distribution scale. By the matrix transformation equation, we can obtain the following equation

$I^{(n)}(l) G=I_{B}^{(n)}$

where $I^{(n)}$ is the row vector distribution of light intensity distribution, $G$ is the column vector representation of objects. In order to describe conveniently, we ignored the twodimensional distribution parameters $x$. In multi-scale ghost imaging system, it need to illuminate multiple scales speckle distribution to the scene and in turn to get the light intensity information by single pixel detector, Although by multi-scale speckle distribution here, we assumes that the speckle size is consistent with the overall distribution. The total process of Nth light intensity information collection, it can be expressed by the available for matrices as follows

$I_{N} G=I_{B N}$

where $I_{N}$ is the multi-scale speckle distribution of the lighting objects. The $s$ line (less than $N$ ) is the s-th to collect the row vector form of the distribution of light speckle, $I_{B N}$ is the column form of intensity distribution obtained by single pixel detector. $I_{B N}=\left[I_{B}^{(1)}, I_{B}^{(2)}, \ldots, I_{B}^{(N)}\right]^{T}, T$ is the matrix transpose operation. Assumes that $K$ is the total number of the inversion image, Traditional evaluation methods require observation at least $\mathrm{K}$ samples can accurately obtain object information recovery. The general object image is sparse under some form of transformation, Which contains a large number of zero elements, Such as discrete cosine transform, Fourier transform and discrete wavelet transform, etc [7-12]. Signal sparse transform can be represented as

$G=\psi \alpha$

where, $\psi$ is sparse matrix. The object of image information $G$ is obtained from solving the sparse signal $\alpha$ using (3) and (4), we have

$\alpha=\arg \min \|\alpha\|$ subject to $I_{N} \psi \alpha=I_{B N}$

For the solution of (5), matching pursuit algorithm, interior-point method and gradient method can be adopted. solving the sparse signal $\alpha$, the object information $G$ can be solved by the sparse transform formula of signal.

Through the analysis of the above, the theory and method of solving the ghost imaging system in this paper is accordance with the conventional theory. The only difference embodied is that this paper restore the information of the object by multiple scales of speckle, This significantly differences can get better results than single scale speckle. It can effectively improve the accuracy of image under the premise of the object image resolution is not affected. In the following simulation research, the experimental results can be confirmed this conclusion.

\section{SIMULATION EXPERIMENT}

The first set of experiments to test object resolution is affected by speckle scale, Every time is fixed in the image restoration speckle scale for recovery, At this time a total of four kinds of circumstances, Corresponding speckle scale to eight pixels, four pixels, two pixels, and a pixel. The total sample number is 300 , sampling rate is seven percent. Fig. (2) shows the original object image and Fig. (3) shows the object image restoration results, $A$ to $D$ speckle scale respectively for $8,4,2$, and 1 pixel cases restored image results. Through this result can obviously get two conclusions: First, the speckle size determines the resolution of the image, the speckle size is smaller, the higher the resolution; Second, the speckle size determines the SNR of the image at the same time, the speckle scale, the greater the image SNR is higher. For the actual demand, we tend to pursuit images of high resolution, At the same time the image is of high SNR. And through the analysis of the above knowable, In a limited number of fixed speckle size sampling, Image resolution and SNR is contradictory, In order to obtain high resolution, image signal-to-noise ratio decreases, whereas the reverse.

The real image has multi-scale resolution, So the multiscale speckle distribution can be used for image restoration, To do so on the one hand could get high resolution images, On the other hand, it can improve the SNR of image. And the resolution of the image is different scales, So in a multiscale ghost imaging system, you need to consider different scales should be possessed by speckle proportion. And the composition of multi-scale speckle can be various, has the very many combinations. So it is impossible to restore the image of each case to carry on the quantitative research, So here we use several has the characteristics of different 


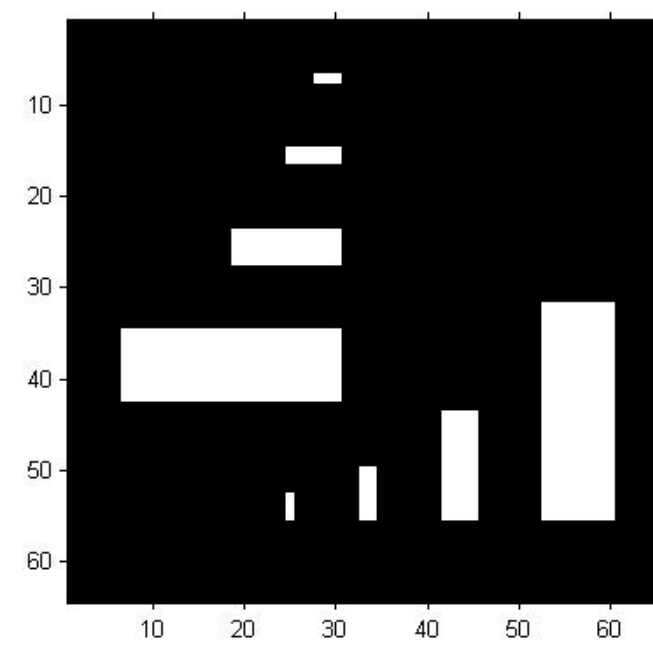

Fig. (2). Original object image.

proportion of speckle scale. Acquisition process to keep the overall sample number is 300 . The quality of the restored image can be introduced to the original judgment error between the real images, Criterion formula is as follows

$E=\sqrt{\left|G_{h}-G_{o}\right|^{2} / K}$
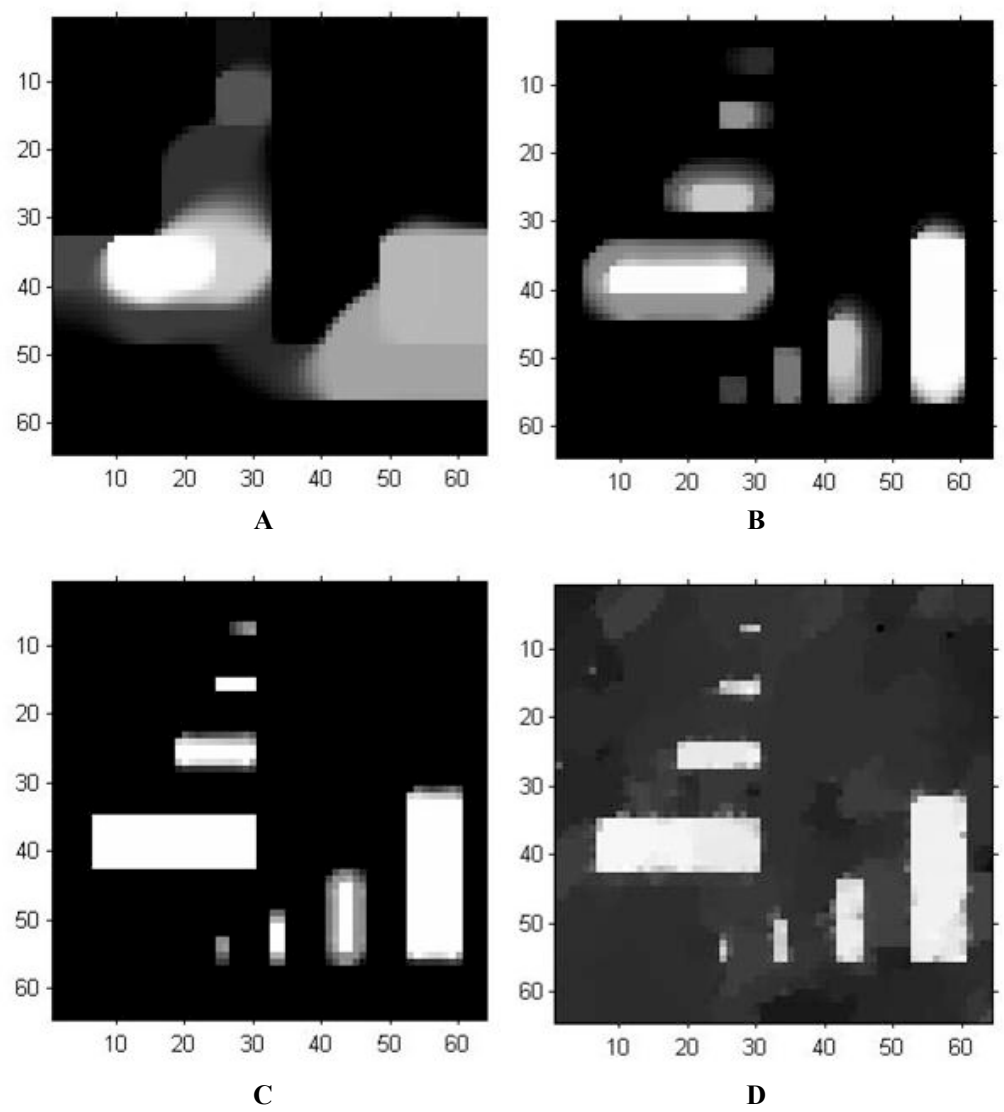

Fig. (3). Results:

A) Restored image in 8 pixel speckles,

B) Restored image in 4 pixel speckles,

C) Restored image in 2 pixel speckles,

D) Restored image in 1 pixel speckles.

where $G_{h}$ is recover image, $G_{o}$ is original image, First give in fixed speckle for minimum resolution (1 px) and four resolution speckle image restoration results under the same proportion, as shows in Fig. (4), where A is Fixed minimum resolution image, The error value is $0.12, \mathrm{~B}$ is proportion of four resolution speckle phase recovery image at the same time. The error value is 0.09 . The experimental results confirm the theoretical analysis results, At the same time it can be seen that due to the small size speckle in sampling number down, leading to a fall in image resolution. The resolution of the images shows the ghost imaging system to recover control, not only by the small size speckle speckle and the small size within the number of sampling density constraints. But at the same time, image error is smaller, that is more close to real images, introduction of multi-scale speckle of healing can get more real images.

The experiments with different proportion of speckle size below, First big speckle size large proportion of cases studies. 8 pixels, 4 pixels, 2 pixels and speckle accounts for more than 1 pixel size is $\left[\begin{array}{llll}0.7 & 0.1 & 0.1 & 0.1\end{array}\right],\left[\begin{array}{llll}0.6 & 0.2 & 0.1 & 0.1\end{array}\right],\left[\begin{array}{ll}0.5 \\ 0.5\end{array}\right.$ $\left.\begin{array}{lll}0.3 & 0.1 & 0.1\end{array}\right]$ and $\left[\begin{array}{llll}0.4 & 0.4 & 0.1 & 0.1\end{array}\right]$. Fig. (5) shows the recovery of computer simulation results, A, B, C and Dis Corresponding to the four cases restored image. The error value of 0.24 , $0.15,0.13$ and 0.1 respectively. The results also confirm the 

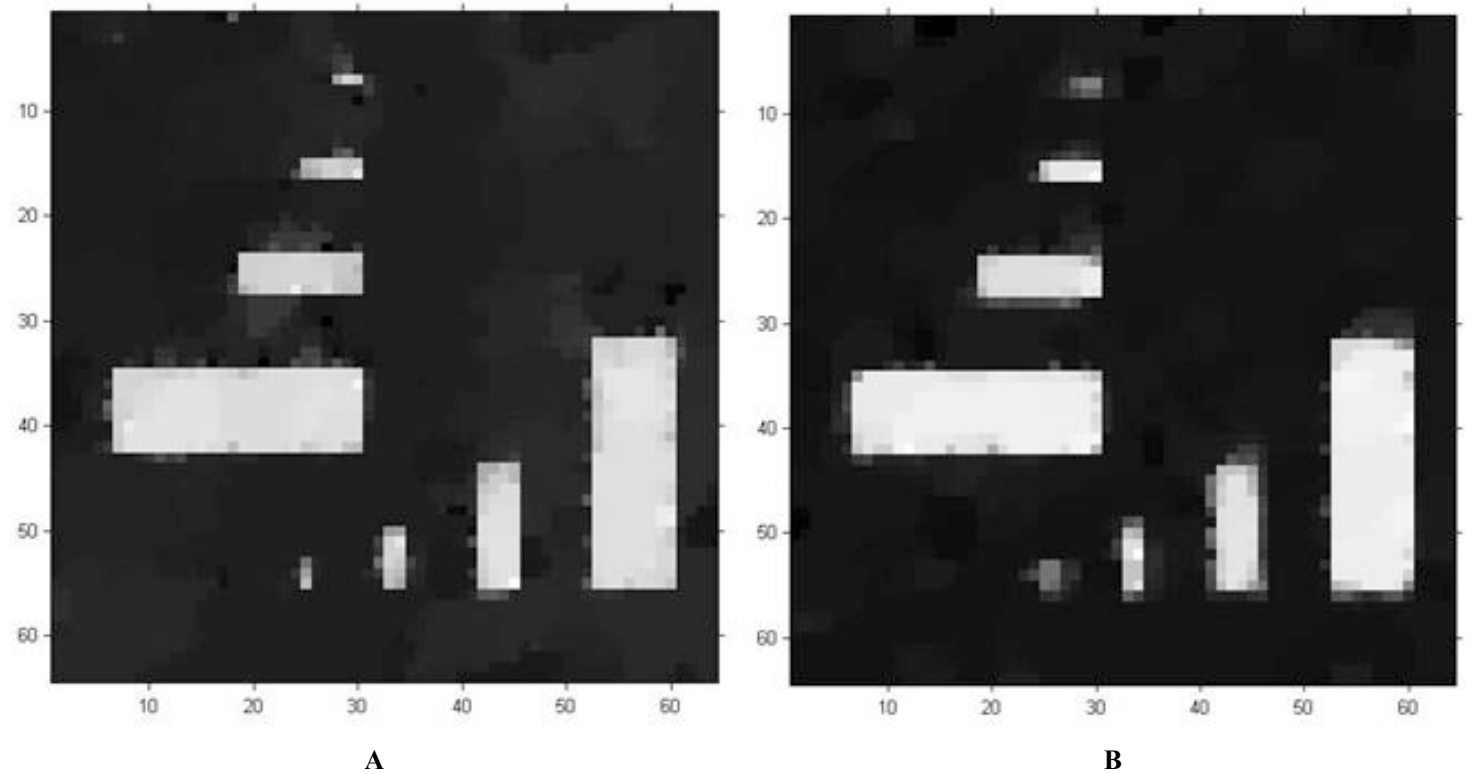

Fig. (4). Results:

A) Restored image in 1 pixel speckles,

B) Restored image in four different pixel and same proportion speckles.
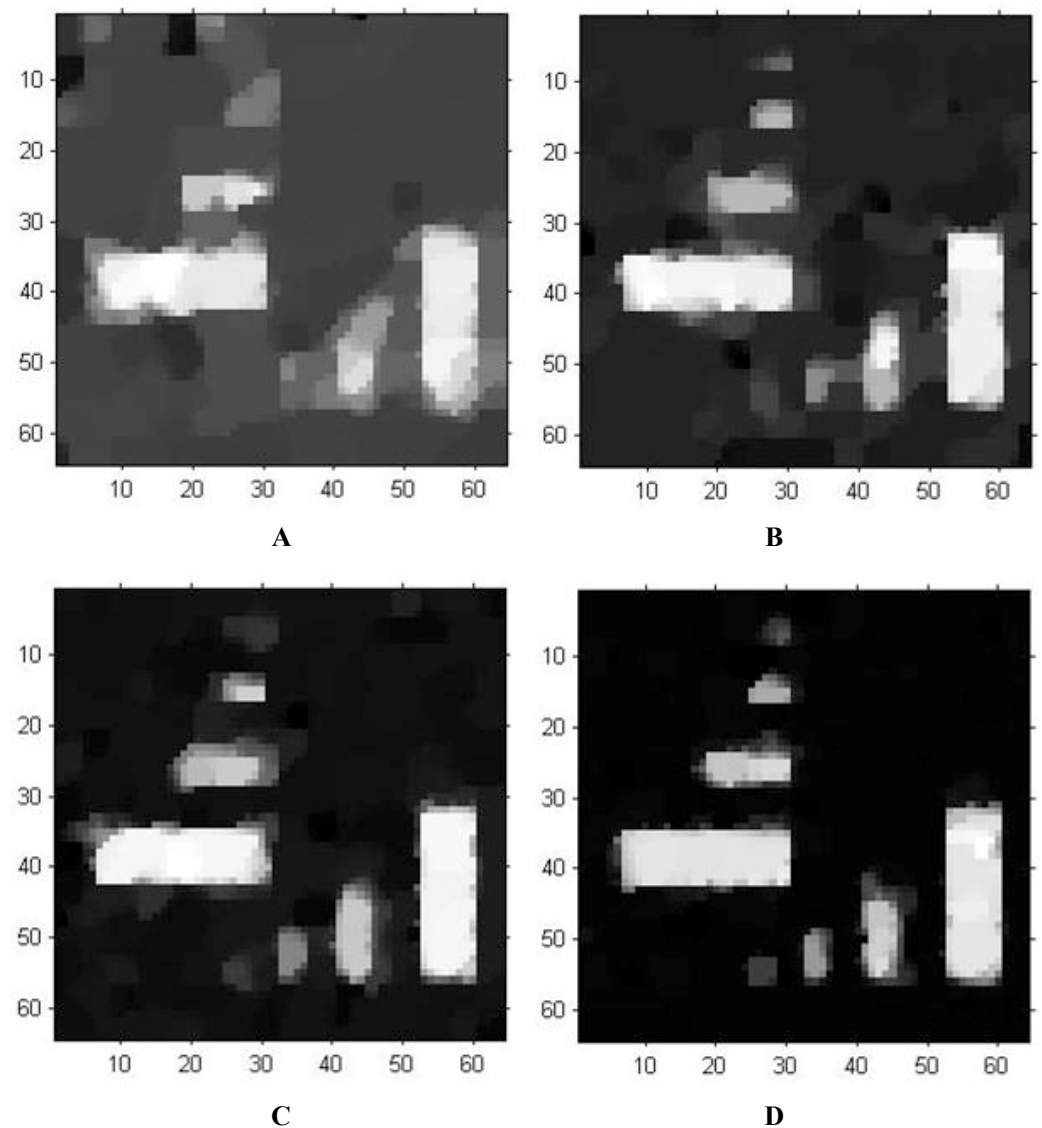

Fig. (5). Results:
A) The first case results,
B) The second case results,
C) The third case results,
D) The fourth case results. 

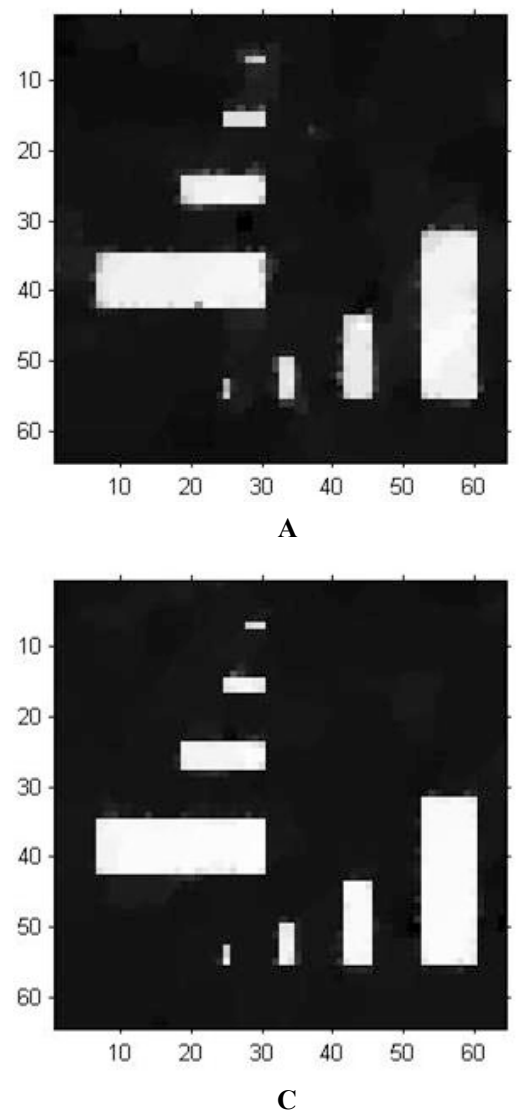

Fig. (6). Results:

A) The first case results,

B) The second case results,

C) The third case results,

D) The fourth case results.

theoretical analysis results, But due to the small size speckle proportion is small, so the resolution of the image is low. But in the case of a large proportion of smaller, can get closer to the real value of the image. Therefore the small speckle size large proportion of cases of recovery. 8 pixels, 4 pixels, 2 pixels and speckle accounts for more than 1 pixel size for 0.1 0.7 [ 0.10 .1$]$, [ [ [ $\left.\begin{array}{llll}0.1 & 0.1 & 0.2 & 0.6\end{array}\right],\left[\begin{array}{llll}0.1 & 0.1 & 0.3 & 0.5\end{array}\right]$ and [ $\left[\begin{array}{ll}0.1 & 0.1\end{array}\right.$

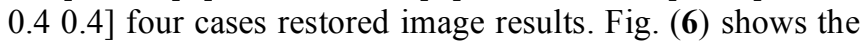
recovery of computer simulation results, A, B, C and D respectively corresponding to the image of the four cases. The error value of $0.07,0.11,0.06$ and 0.08 respectively. As can be seen in the small size speckle occupied large proportion of cases, to obtain the image results are better than the image of the fixed small speckle size is more close to the real images, And no change at this time the resolution of the image, and the big size large proportion of different resolution decreased. According to the above two groups of contrast test, you can see that the sampling multi-scale speckle in image restoration can get close to the real value of the image, And occupies a large proportion of cases in small scale to get the image more close to the real value, and with the resolution of the fixed small speckle size.

Through the above simulation results, can be obtained using multi-scale speckle image restoration can get closer to the true value of the image, And small scale speckle occupies
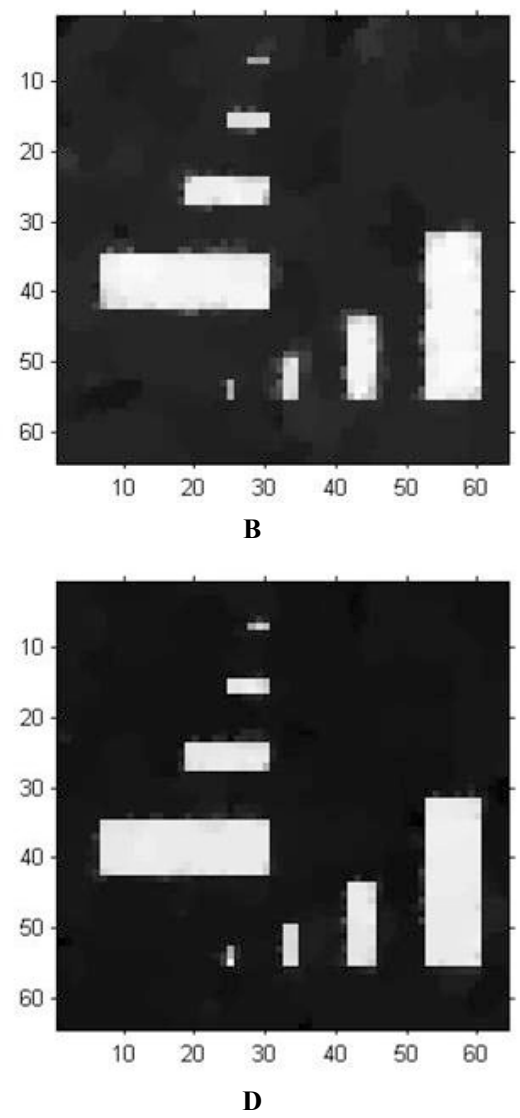

large proportion can get high resolution image. Multi-scale compression perception ghost imaging system with fixed size ghost imaging system has the advantages of high resolution and high reliability. Due to the diversity of the speckle size and the diversity of different size image resolution, the simulation is only qualitative analysis results are given.

\section{CONCLUSION}

Existing ghost imaging system using a single speckle scale for image restoration, While the existing precision optical modulator can implement multi-scale speckle. In this paper, based on the multi-scale speckle ghost imaging system is studied, During the irradiation of objects with multiple dimensions of speckle illumination and acquisition object reflected light is strong, Using the intensity information obtained from the modulation and information acquisition, the compression algorithm for recovery of object image perception. Simulation experiments using has four different scale image resolution were studied. Results show that the multiscale compression perception ghost imaging system than single fixed scale ghost imaging system can be more close to the real value of the image, And discern don't change. The method to the real ghost imaging system has the reference value, can be widely used in the existing ghost imaging system, get more real object image. 


\section{CONFLICT OF INTEREST}

The authors confirm that this article content has no conflict of interest.

\section{ACKNOWLEDGEMENTS}

Zhang na is supported by NSFC (No.61202248).

\section{REFERENCES}

[1] J. H. Shapiro, and R. W. Boyd, "The physics of ghost imaging", Quantum Information Processing, vol. 11, pp. 949-993, 2012.

[2] B. Sun, M.P. Edgar, R. Bowman, L. E. Vittert, S. Welsh, A. Bowman, and M. J. Padgett, "3D Computational Imaging with SinglePixel Detectors," Science, vol. 340, no. 6134, pp. 844-7, 2013.

[3] D. Shi, S. Hu, and Y. Wang, "Polarimetric ghost imaging", Optics Letters, vol. 39, pp. 1231-1234, 2014.

[4] G.A. Howland, D.J. Lum, M.R. Ware, and J.C. Howell, "Photon counting compressive depth mapping," Optics Express, vol. 21, pp. 23822-23837, 2013.

[5] W. Gong, C. Zhao, J. Jiao, E. Li, M. Chen, H. Wang, W. Xu, and S. Han, "Three-dimensional ghost imaging ladar", arXiv: 1301. 5767,
2013.

[6] E. Zhang, and H. Dai, "Effect of light polarization on thermal light correlated imaging", Acta Physica Sinica, vol. 60, no. 6, pp. 206213, 2011.

[7] S. Zhang, J. Wang, J. Wang, H. Li, and X. Liu, "Simple calculation method for three-dimensional imaging based on compressed sensing", Acta Optica Sinica, vol. 1, pp. 84-90, 2013.

[8] Y. Ma, Y. Wang, Y. Wang, M. Ge, Y. Wang, H. Qi, and R. Shu, "Study of single-pixel detection computational imaging technology based on compressive sensing," Acta Optica Sinica, vol. 33, no. 12, pp. 11-13, 2013.

[9] J. Chen, and Y. Wang, "Research of the Compressive Imaging Technology," Laser \& Optoelectronics Progress, vol. 2012, no. 3, pp. 15-22, 2012.

[10] X. Li, and B. Li, "Review on progress of real-time $\mathrm{THz}$ sensing and imaging technology," Laser \& Optoelectronics Progress, vol. 2012, no. 9, pp. 55-60, 2012.

[11] E.J. Candes, J. Romberg, and T. Tao, "Robust uncertainty principles: Exact signal reconstruction from highly incomplete frequency information," IEEE Transactions on Information Theory, vol. 52, no. 2, pp. 489-509, 2006

[12] W. Shao, and Z. Wei, "Advances and perspectives on compressed sensing theory," Journal of Image and Graphics, vol. 1, pp. 1-12, 2012 .

Received: September 22, 2014

Revised: November 30, 2014

Accepted: December 02, 2014

(C) Na and Quan; Licensee Bentham Open.

This is an open access article licensed under the terms of the Creative Commons Attribution Non-Commercial License (http://creativecommons.org/licenses/by-nc/3.0/) which permits unrestricted, non-commercial use, distribution and reproduction in any medium, provided the work is properly cited. 